\title{
Quantifying the effect of biopsy lateral decubitus patient positioning compared to supine prostate MRI scanning on prostate translocation and distortion
}

Žiga Snoj*1,2,3; Leonardo Rundo*1,4 ; Andrew B. Gill ${ }^{1,5}$; Tristan Barrett ${ }^{1,6}$

${ }^{1}$ Department of Radiology, Addenbrooke's Hospital and University of Cambridge, Cambridge, United Kingdom; ${ }^{2}$ Radiology Institute, University Medical Centre Ljubljana, Ljubljana, Slovenia; ${ }^{3}$ Medical Faculty, University of Ljubljana, Ljubljana, Slovenia; ${ }^{4}$ Cancer Research UK Cambridge Centre, Cambridge, United Kingdom;

${ }^{5}$ Department of Medical Physics, Cambridge University Hospitals, Cambridge, United Kingdom; ${ }^{6}$ CamPARI Clinic, Addenbrooke's Hospital and University of Cambridge, Cambridge, United Kingdom

*Equal contributors

Acknowledgements: The authors acknowledge research support from Cancer Research UK, Prostate Cancer UK, National Institute of Health Research Cambridge Biomedical Research Centre, Cancer Research UK and the Engineering and Physical Sciences Research Council Imaging Centre in Cambridge and Manchester and the Cambridge Experimental Cancer Medicine Centre, Addenbrooke's Charitable Trust, the National Institute for Health Research (NIHR) Cambridge Biomedical Research, Cambridge University Hospitals NHS Foundation Trust.

Cite as: Can Urol Assoc J 2020 March 30; Epub ahead of print. http://dx.doi.org/10.5489/cuaj.6298

Published online March 30, 2020

$* * *$

\section{Abstract}

Introduction: More than a quarter of tumors are missed by magnetic resonance imaging/ultrasound (MRI/US) fusion-guided biopsy, the majority due to software-based misregistration. Transrectal approaches to biopsy are typically performed in the lateral decubitus position; conversely, diagnostic MRI is performed with the patient lying supine. Any positionrelated difference in prostate location or gland deformation could potentially exacerbate misregistration at subsequent biopsy.

Methods: Fifteen healthy male volunteers (mean age 35.9 years, range 27-53) were included in this prospective, institutional review board-approved study. Each volunteer had an MRI performed in the supine position, followed by the second in the lateral decubitus position (mimicking a typical biopsy position). MRI images were co-registered and analyzed in order to assess prostate translocation and distortion.

Results: Whole prostate translocation of $\geq 5 \mathrm{~mm}$ was observed in $20 \%$ of patients and $\geq 3 \mathrm{~mm}$ in $60 \%$ of patients. When dividing the prostate into prostatic sectors, the prostatic base 
demonstrated the largest positional difference. When plotting the translocation directions with relative volume difference, there was a moderate negative correlation trend in the latero-lateral direction. Only minimal distortion was observed, with similar distortion among all prostatic sectors.

Conclusions: Positional change affects the prostate translocation, however, the effect on prostate distortion appears to be negligible. Prostate translocation in latero-lateral direction can be minimized with larger bladder volumes. Thereby, prostate translocation needs to be considered alongside software misregistration error; however, positional change should not affect software registration of MRI/US fusion-guided prostate biopsy.

\section{Introduction}

Prostate cancer $(\mathrm{PCa})$ is the commonest male non-cutaneous cancer worldwide, with its incidence continuing to increase due to an ageing population. ${ }^{1,2}$ The traditional diagnostic workup with systematic transrectal ultrasound (TRUS) guided biopsy of the gland is limited, missing up to $50 \%$ of tumours and under-grading approximately a third. ${ }^{3,4,5}$ Magnetic resonance imaging (MRI) has high sensitivity for prostate cancer but poor specificity and therefore needs to be supplemented by biopsy. ${ }^{4}$ As a result, recent Level 1 evidence supports the use of initial multiparametric (mp) MRI in order to target subsequent biopsy as the optimal rule in test for clinically significant $\mathrm{PCa}$. , $6,7,8$

Targeted biopsy can be performed as an in-gantry MRI-guided procedure, which requires MRI compatible equipment and scanner availability, cognitive, which is typically operator and experience dependent, or using fused MRI/US techniques. The second approach combines the advantages of MRI for lesion identification and US for real-time biopsy acquisition in the outpatient setting and has consistently shown superior cancer detection rates compared to systematic 12-core TRUS biopsy. 9,10

Target biopsy alone is appealing in order to reduce morbidity, and can help reduce the detection of clinically insignificant cancer. ${ }^{7,11,12}$ However, several studies have shown systematic background biopsy detects additional tumours missed with targeted cores alone. ${ }^{13}$ Increasing the number of cores surrounding the target either by a "focal saturation" approach or adding "ipsilateral only" systematic biopsies to targeted cores has been shown to increase the detection of clinically significant cancer detection. ${ }^{8,14,15}$ These results imply a targeting error either due to the fusion software or the operator. Furthermore, a recent study showed that among the $27 \%$ of tumours missed by MRI/US fusion-guided biopsy, the majority were due to software misregistration. ${ }^{9}$ Transrectal approaches to biopsy are typically performed in the lateral decubitus position, ${ }^{16}$ conversely diagnostic MRI is performed with the patient lying supine. Any positionrelated difference in prostate location or gland deformation could potentially exacerbate mis- 
registration at subsequent biopsy, particularly if a rigid rather than elastic fusion platform is used. ${ }^{17}$ To our knowledge, such differences have never been assessed, therefore, the aim of our study was to quantify any changes in prostate location or shape in relation to patient positioning

\section{Methods}

15 healthy male volunteers (mean age 35.9 years, median 34, range 27-53) were included in this prospective, institutional review board-approved study (Ethics Reference Anonymised), with all participants signing written informed consent.

\section{Magnetic resonance imaging}

For each study, two MRI scans were acquired. Prior to scanning, patients were instructed to empty their bladder in order to reduce any confounding effects of differing bladder volumes on prostate position. Immediately after the first MRI scan had been performed in the supine position, the patients were instructed to change to the lateral decubitus position (close to fetal position), mimicking that of a typical biopsy in order to acquire the second MRI scan. MRI was performed on a 3T MR750 magnet (General Electric Healthcare, Waukesha, WI, USA) using a 32-channel phased-array body coil. Sequences included: high-resolution axial T2weighted (T2w) fast recovery fast spin echo (FRFSE) imaging, TR/TE of 3663/102 ms field-ofview (FOV) $22 \times 22 \mathrm{~cm}^{2}, 3 \mathrm{~mm}$ slice thickness with no gap, in-plane resolution $0.85 \times 0.57 \mathrm{~mm}$, and 3 signal averages; sagittal T2 $\mathrm{w}$ cube sequence FOV $22 \times 22 \mathrm{~cm}^{2}, 1 \mathrm{~mm}$ slice thickness with no inter-slice gap, in-plane pixel size $1.0 \times 0.8 \mathrm{~mm}^{2}$.

\section{Image analysis}

Figure 1 schematises the overall workflow. Figure 1a depicts the first phase of our procedure for quantitatively evaluating prostate translocation and distortion. For each study, the co-registration of the two MRI data sets was manually performed by a board-certified radiologist using ITKSNAP in the sagittal plane by exploiting bony landmarks (i.e., lumbar spine and pelvic bones). ${ }^{18}$ ITK-SNAP is a medical image processing tool that exploits the $\mathrm{C}++$ Insight Toolkit (ITK) library. ${ }^{19}$ The affine transformation matrix (including rigid-body transformations as well as scaling to take into account different field of views) was then applied by means of Advanced Normalisation Tools (ANTs). ${ }^{20,21}$ More precisely, the lateral decubitus scan ('moving' volume) was co-registered against the corresponding supine scan ('fixed' volume). Each co-registered image was then reformatted in the axial plane to allow for a more accurate and clinically relevant prostate delineation. Using a custom software tool, the outlines were drawn around the prostatic tissue from the most inferior to the most superior point where the prostatic tissue could be clearly identified, excluding seminal vesicles, with reference to the separately acquired high-resolution T2w FRFSE axial images. The outlining procedure was performed in consensus with a boardcertified uro-radiologist with 8-years' experience in reporting prostate MRI. 
In our analysis, a prostate subdivision into prostatic sectors (i.e., apex, mid-gland, and base) was also considered by dividing prostate into thirds. Moreover, for better appreciating the distortion directions in the axial section, a subdivision of the prostate into four quadrants was performed. A more detailed explanation of the computerised analysis is provided in Supplementary Material. Briefly, relying on a computational method previously devised and validated for prostate deformation assessment, ${ }^{22}$ the two prostate glands under investigation are translated aligned to their centres-of-mass, then the slice delineations of the 'moving' volume were translated onto the 'fixed' image space (Figure 1b). According to Figure 1c, we computed the 'resultant translocation' $t$ to characterise the global translocation of the prostate. The Root Mean Square (RMS) value of the magnitude of the resultant translocation vector by averaging over all the slices was then calculated. Lastly, the 'resultant distortion' $d$ to assess the combined effects of translational and local distortions (Figure 1d). Aiming at a graphical and intuitive representation, two examples of distortion maps, along with the corresponding fixed and moving volumes, are depicted in Figure 2.

\section{Bladder volume and rectum distention assessment}

Bladder and rectal volumes are potential confounders that may alter prostate position. Bladder volumes were calculated via whole volume segmentation on sagittal T2w cube sequence using an in-house software developed in MatLab (MathWorks, Natick, MA, USA). ${ }^{23}$ Rectal distention was derived by using the maximum sagittal and axial dimensions (i.e., anal canal to peritoneal reflection), and subjectively using a 5-point Likert scale following a previously described Likert scale. $^{24}$

\section{Group design}

The quality of the lateral decubitus studies was subjectively evaluated, and in 5 cases image quality was insufficient to make accurate co-registration or prostate outlines; these cases were excluded from further analysis.

\section{Intra-observer reliability}

Single observer prostate outlining was performed in all cases. After the primary prostate outlining was performed, in a sub-set of 5 studies, prostate outlining was performed again by the same observer in order to assess the intra-observer repeatability.

\section{Statistics}

Continuous variables were expressed as mean \pm standard deviation (SD). Pearson correlation coefficient $(r)$ was calculated to evaluate correlation. Significance was set at $p<0.05$. Statistical analysis was performed with SPSS v.17.0 (SPSS Inc., Chicago, Illinois, USA). 


\section{Results}

\section{Prostate translocation}

Supine post-void bladder volume was $85.1 \pm 51.1 \mathrm{ml}$ (range: $41.1-202.5$ ), and lateral decubitus post-void bladder volume was $125.8 \pm 60.4 \mathrm{ml}$ (range: $65.1-217.0$ ). The absolute difference in bladder volume change was $40.6 \pm 30.0 \mathrm{ml}$ (range: $13.2-116.9$ ). Prostate volume was $32.2 \pm$ $10.4 \mathrm{ml}$ (range: $21.8-50.1$ ). The median value for rectal distension was 3 (range: $1-5$ ). The average absolute difference in rectal change was $20.5 \pm 13.6 \mathrm{ml}$ (range: $4.2-48.2$ ).

Whole prostate translocation of $\geq 5 \mathrm{~mm}$ was observed in $2 / 10$ patients and $\geq 3 \mathrm{~mm}$ in $6 / 10$ patients. When dividing the prostate into base, mid-gland and apex, the prostatic base showed the largest positional difference (Figure 3). Base translocation of $\geq 5 \mathrm{~mm}$ was observed in 3/10 patients and of $\geq 3 \mathrm{~mm}$ in $7 / 10$ patients. The prostatic translocation directions are shown in Figure 3. When plotting the translocation directions with relative volume difference, there was a moderate negative correlation trend in the latero-lateral (LL) direction $(r=-0.59, p=0.07)$; a significant moderate positive correlation was observed in the antero-posterior (AP) direction $(r=0.74, p=0.01)$; with a weak and insignificant correlation observed in the supero-inferior (SI) direction $(r=30, p=0.40)$ (Figure 4$)$.

\section{Prostate distortion}

Only minimal distortion was observed (Table 1). Similar distortion was observed among all prostatic sectors. Furthermore, no differences were observed when sub-dividing the prostate into anterior, posterior, left and right (Table 1).

\section{Intra-observer reliability}

Good reproducibility was observed with only minimal differences. The reliability measurements for the translocation direction were $0.01 \pm 0.09 \mathrm{~mm}$ (range: $0.01-0.27$ ) in the LL direction; 0.09 $\pm 0.09 \mathrm{~mm}$ (range: $0.03-0.19$ ) in the AP direction and $0.03 \pm 0.02 \mathrm{~mm}$ (range: $0.00-0.05$ ) in the SI direction. The intra-observer reproducibility measurements for prostatic sectors are depicted in Table 2.

\section{Discussion}

The results of our study suggest that positional change can affect the prostate translocation in latero-lateral direction, however, the effect on prostate distortion appears to be negligible. Furthermore, it appears that prostate translocation with positional change may be minimised with larger bladder volumes.

To the best of our knowledge, the effect of positional change on prostate translocation has not been previously described. Studies to date that relate to patient positioning during biopsy have typically focused on patient satisfaction rather than biopsy accuracy, with the lateral 
decubitus position proving to be most tolerable. ${ }^{25}$ However, Halpern et al. investigated the effect of patient position using Doppler US and demonstrated an increased blood flow in the depended side of the prostate, when changing position from supine to the lateral decubitus position. ${ }^{26}$ The effect of position was studied in only three patients, but in combination with our study it shows that patient positioning may be an important consideration in prostate imaging. ${ }^{27}$ The amplitude of prostate translocation in our study was similar to that of previous studies evaluating the effect of bladder filling and rectal loading on prostate position. ${ }^{27,28}$ Nevertheless, there are notable differences in the direction of prostate translocation, which was mainly in the AP direction in these studies, with LL translocation being negligible. ${ }^{26,28}$ In our study, a different pattern was observed with prominent translocation being seen in the LL direction. The translocation was gravity-dependent, a similar effect to Halpern et al. study. ${ }^{26}$ LL translocation may have an important impact in everyday clinical practice as transrectal prostatic biopsy is typically performed in the left lateral decubitus position, whereas diagnostic MR images are acquired with the patient lying supine. This may especially be the case when MRI/US fusion-guided biopsy is performed, and could add to mis-registration error due to translocation or deformation of the targeted tissue. ${ }^{9}$

A prostate tumour is considered to be clinically significant if it has a volume of at least $0.5 \mathrm{ml} .{ }^{29}$ Karnik et al. claim that RMS of $2.5 \mathrm{~mm}$ yields a probability of $95.4 \%$ that the registered targets will lie inside the clinically significant $5 \mathrm{~mm}$ radius. ${ }^{30}$ This calculation is only valid if there are no additional sources of error in the clinical application. ${ }^{31}$ According to previous studies, the observed RMS of prostate translocation of nearly $4 \mathrm{~mm}$ in our study would correspond to a $82 \%$ probability of hitting a target. ${ }^{31}$ This is of low importance in MRI/US fusion-guided biopsies since prostate translocation can be compensated for with fusion software (the outline being offset, but the prostate shape maintained), but is an important finding that needs to be acknowledged by an operator performing cognitive biopsies. To perform an accurate cognitive biopsy, the operator needs to have a good understanding of the lesion position in the prostate and the background knowledge of the prostate translocation with positional change may aid in planning the biopsy. ${ }^{32}$

Prostate distortion cannot be compensated with fusion software and it may impact cognitive guidance. Multiple MRI/US software platforms are available allowing either a rigid or elastic image registration.32 In our study rigid transformation was used with only minimal distortion of prostatic tissue observed for whole gland as well as between base, mid-gland and apex, and no differences were observed when the prostate was divided into anterior, posterior, right and left quadrants. It has to be noted that in our study only the effect of positioning was studied and this is likely negligible compared to that induced by the placement of a rectal ultrasound probe during biopsy. Delongchamps et al. compared rigid and elastic image registration and showed a non-significant increase in PCa detection rate in favour of the elastic system - any improvement may be attributed to better compensation of the rectal probe 
distortion. ${ }^{33} \mathrm{We}$ accounted for the confounding factors of bladder and rectal volume, as these issues have long been recognised in radiotherapy, which typically employs dedicated preparation protocols in clinical practice to mitigate their impact. ${ }^{34}$ Although rectal loading may be less of an issue with an endorectal probe being for transrectal prostate biopsy, the procedure does not routinely incorporate any bladder preparation protocol. Bladder volume effect has been previously investigated in three patients by Lofti et al. who concluded that bladder filling has a negligible effect on the LL translocation of prostate in either the lateral decubitus or supine positions. ${ }^{27}$ In our study MRI was performed post-void, thus patients had relatively empty bladders to minimise this as a confounding factor. However, some degree of bladder filling was observed between the two MRI scans. When plotting relative volume difference against the direction of translation, two interesting observations were made. Firstly, the observed positive correlation in AP direction; this is expected, due to the previously established association of bladder filling and AP translocation. ${ }^{27,28}$ Secondly, with bladder larger volume differences there was a moderate negative trend towards lower translocation observed in the LL direction, thus the greater the bladder volume, the more fixed the prostate appears to be. This may be explained by the direct contact of the bladder to the prostatic base, thus with bladder filling there would be less potential space for prostate translocation.

Consistently with other studies, the base of the prostate was shown to have a larger amplitude of translocation than the apex, presumably due to apex being relatively fixed by the pelvic musculature. ${ }^{27,35}$ Of note, Iremashvili et al. described a decreased biopsy accuracy in the lateral mid- and basal cores. ${ }^{36}$ This fact may be interpreted in the context of different PCa spatial frequencies, with previous studies having shown an increased incidence of $\mathrm{PCa}$ in the apex and a correspondingly low incidence in the base: ${ }^{37}$ however, the relative mobility of the prostatic base may be an important factor to be considered, especially with smaller sized targets.

Our study has some limitations. First, the recruited subjects were young healthy individuals and may not be representative of the patient population seen in everyday clinical practice. However, it is possible that the amplitudes of the LL prostate translocation may be even more pronounced in clinical practice. Second, the group sample is rather small, but this is due to the very specific research question, and future studies with a higher number of patients are needed in order to fully understand the mechanism of prostate translocation due to patient positioning.

\section{Conclusions}

In conclusion, positional change affects the prostate translocation, however the effect on prostate distortion appears to be negligible. Prostate translocation in the LL direction can be minimized with larger bladder volumes. Prostate translocation needs to be considered alongside software mis-registration error, however positional change should not affect software registration of MRI/US fusion-guided prostate biopsy. 


\section{References}

1. Siegel RL, Miller KD, Jemal A. Cancer Statistics, 2019. CA Cancer J Clin 2019;69(1):734.

2. Center $\mathrm{MM}$, Jemal $\mathrm{A}$, Lortet-Tieulent $\mathbf{J}$ et al. International variation in prostate cancer incidence and mortality rates. Eur Urol 2012;61(6):1079-1092.

3. Kvåle R, Møller B, Wahlqvist R et al. Concordance between Gleason scores of needle biopsies and radical prostatectomy specimens: a population-based study. BJU Int 2009;103(12):1647-1654.

4. Ahmed HU, El-ShaterBosaily A, Brown LC et al. Diagnostic accuracy of multiparametric MRI and TRUS biopsy in prostate cancer (PROMIS): a paired validating confirmatory study. Lancet 2017;389:815-822.

5. Serefoglu EC, Altinova S, Ugras NS, Akincioglu E, Asil E, Balbay MD. How reliable is 12-core prostate biopsy procedure in the detection of prostate cancer?. Can Urol Assoc J 2013;7(5-6):293-298.

6. Rouvière $\mathrm{O}$, Puech $\mathrm{P}$, Renard-Penna $\mathrm{R}$ et al. Use of prostate systematic and targeted biopsy on the basis of multiparametric MRI in biopsy-naive patients (MRI-FIRST): a prospective, multicentre, paired diagnostic study. Lancet Oncol 2019;20(1):100-109.

7. Kasivisvanathan V, Rannikko AS, Borghi M et al. MRI-targeted or standard biopsy for prostate-cancer diagnosis. N Engl J Med 2018;378:1767-1777

8. van der Leest M, Cornel E, Israël B et al. Head-to-head comparison of transrectal ultrasound-guided prostate biopsy versus multiparametric prostate resonance imaging with subsequent magnetic resonance-guided biopsy in biopsy-naive men with elevated prostate-specific antigen: a large prospective multicenter clinical study. Eur Urol 2019;75(4):570-578.

9. Coker MA, Glaser ZA, Gordetsky JB, Thomas JV, Rais-Bahrami S. Targets missed: predictors of MRI-targeted biopsy failing to accurately localize prostate cancer found on systematic biopsy. Prostate Cancer Prostatic Dis 2018;21(4):549-555.

10. Mendhiratta N, Meng X, Rosenkrantz AB et al. Prebiopsy MRI and MRI-ultrasound fusion-targeted prostate biopsy in men with previous negative biopsies: impact on repeat biopsy strategies. Urology 2015;86(6):1192-1198.

11. Pepe P, Aragona F. Morbidity after transperineal prostate biopsy in 3000 patients undergoing 12 vs 18 vs more than 24 needle cores. Urology 2013;81:1142-1146.

12. Papagiannopoulos D, Abern M, Wilson N, O'Block N, Raff L, Coogan C, Latchamsetty KC. Predictors of Infectious Complications after Targeted Prophylaxis for Prostate Needle Biopsy. J Urol 2018;199:155-160.

13. Lu AJ, Syed JS, Ghabili K et al. Role of core number and location in targeted magnetic resonance imaging-ultrasound fusion prostate biopsy. Eur Urol 2019;76(1):14-17.

14. Bryk DJ, Llukani E, Taneja SS, Rosenkrantz AB, Huang WC, Lepor H. The role of ipsilateral and contralateral transrectal ultrasound-guided systematic prostate biopsy in men with unilateral magnetic resonance imaging lesion undergoing magnetic resonance imaging-ultrasound fusion-targeted prostate biopsy. Urology 2017;102:178-182. 
15. Nienke NL, Barrett T, Lloyd T, Warren AY, Samel C, Bratt O, Kastner C. Optimizing the number of cores for MRI guided targeted and systematic transperineal prostate biopsy. BJU Int. 2019. doi: 10.1111/bju.14865. [Epub ahead of print]

16. Spirnak JP, Resnick MI. Ultrasound. In: Adult \& Pediatric Urology. Gillenwater JY, Grayhack JT, Howards SS, Mitchell ME. Philadelphia: Lippincott Williams \& Wilkins; 2002;140.

17. Das CJ, Razik A, Sharma S, Verma S. Prostate biopsy: when and how to perform. Clin Radiol 2019. doi: 10.1.17/j.crad.2019.03.016. [In press]

18. Yushkevich, P. A., Gao, Y., Gerig, G. ITK-SNAP: an interactive tool for semi-automatic segmentation of multi-modality biomedical images. In Proc. 38th Annual International Conference of the IEEE Engineering in Medicine and Biology Society (EMBS). 2016; pp. 3342-3345.

19. Yoo, T. S., Ackerman, M. J. Open source software for medical image processing and visualization. Comm ACM 2005;48(2),55-59.

20. Avants BB, Tustison NJ, Stauffer M, Song G, Wu B, Gee JC. The Insight ToolKit image registration framework. Front Neuroinform 2014;8:44.

21. Tustison NJ, Cook PA, Klein A et al. Large-scale evaluation of ANTs and Free Surfer cortical thickness measurements. NeuroImage 2014;99:166-79.

22. Gill AB, Czarniecki M, Gallagher F.A., Barrett T. A method for mapping and quantifying whole organ diffusion-weighted image distortion in MR imaging of the prostate. Sci Rep 2017;7(1):12727.

23. Sushentsev N, Tanner J, Slough RA, Kozlov V, Gill AB, Barrett T. The effect of different drinking and voiding preparations on magnetic resonance imaging bladder distention in normal volunteers and patients. Can Assoc Radiol J 2018;69(4):383-389.

24. Caglic I, Hansen NL, Slough RA, Patterson AJ, Barrett T. Evaluating the effect of rectal distension on prostate multiparametric MRI image quality. Eur J Radiol 2017;90:174180.

25. Lodeta B, Lodeta M. Prostate biopsy in the left lateral decubitus position is less painful than prostate biopsy in the lithotomy position: a randomized controlled trial. Korean $J$ Urol 2012;53(2):87-91.

26. Halpern EJ, Frauscher F, Forsberg F et al. High-frequency Doppler US of the prostate: effect of patient position. Radiology 2002;222(3):634-639.

27. Lotfi M, Bagheri MH, Mosleh-Shirazi MA, Faghihi R, Baradaran-Ghahfarokhi M. Evaluation of the changes in the shape and location of the prostate and pelvic organs due to bladder filling and rectal distension. Iran Red Crescent Med J 2011;13(8):566-575.

28. Padhani AR, Khoo VS, Suckling J et al. Evaluating the effect of rectal distension and rectal movement on prostate gland position using cine MRI. Int J Radiat Oncol Biol Phys 1999;44(3):525-533.

29. Epstein JI, Sanderson H, Carter HB, Scharfstein DO. Utility of saturation biopsy to predict insignificant cancer at radical prostatectomy. Urology 2005;66(2):356-360.

30. Karnik VV, Fenster A, Bax J et al. Assessment of image registration accuracy in three dimensional transrectal ultrasound guided prostate biopsy. Med Phys 2010;37(2):802813. 
31. Baumann M, Mozer P, Daanen V, Troccaz J. Prostate biopsy tracking with deformation estimation. Med Image Anal 2011;16 (3):562-576.

32. Logan JK, Rais-Bahrami S, Turkbey B et al. Current status of magnetic resonance imaging (MRI) and ultrasonography fusion software platforms for guidance of prostate biopsies. BJU Int 2014;114:641-652.

33. Delongchamps NB, Peyromaure M, Schull A, et al. Prebiopsy magnetic resonance imaging and prostate cancer detection: comparison of random and targeted biopsies. $J$ Urol 2013;189:493-499.

34. Maggio A, Gabriele D, Garibaldi E, et al. Impact of a rectal and bladder preparation protocol on prostate cancer outcome in patients treated with external beam radiotherapy. Strahlenther Onkol 2017;193(9):722-732.

35. Wu J, Haycocks T, Alasti $\mathrm{H}$ et al. Positioning errors and prostate motion during conformal prostate radiotherapy using on-line isocentre set-up verification and implanted prostate markers. Radiother Oncol 2001;61(2):127-133.

36. Iremashvili V, Pelaez L, Jorda M et al. Prostate sampling by 12-core biopsy: comparison of the biopsy results with tumor location in prostatectomy specimens. Urology 2012;79(1):37-42.

37. Sazuka T, Imamoto T, Namekawa T et al. Analysis of preoperative detection for apex prostate cancer by transrectalbiopsy. Prostate Cancer 2013;2013:705865.

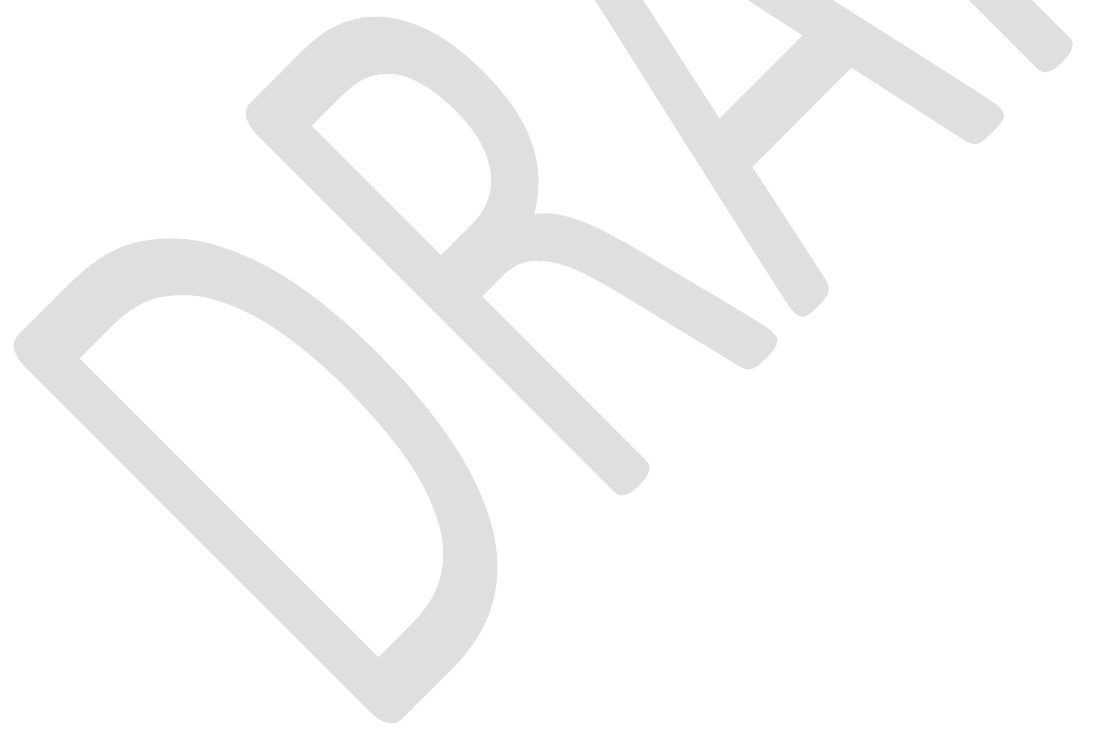




\section{Figures and Tables}

Fig. 1. Overall scheme of the computerized image analysis. (a) $3 \mathrm{D}$ affine co-registration of the lateral decubitus scan ('moving' volume) against the sagittal supine scan ('fixed' volume).

Subsequent manual delineation of the prostate on the two scans by using the axial reformatting. $3 \mathrm{D}$ rigid-body (translation alone $\mathbf{t}$ ) volume alignment between the centres-of-mass of the two prostate glands under investigation. (b) For each slice, the volume sections are aligned so that their centroids are coincident (information stored in the 'tree' of slice centroid translations $\mathbf{T}_{\mathrm{s}}$ ).

(c) Calculation of the RMS of the resultant translocation vector $\mathbf{t}_{\text {res. }}$ (d) Calculation of the resultant distortion vector $\mathbf{d}_{\text {res, }}$ by considering also the subdivision of the axial plane into four quadrants.

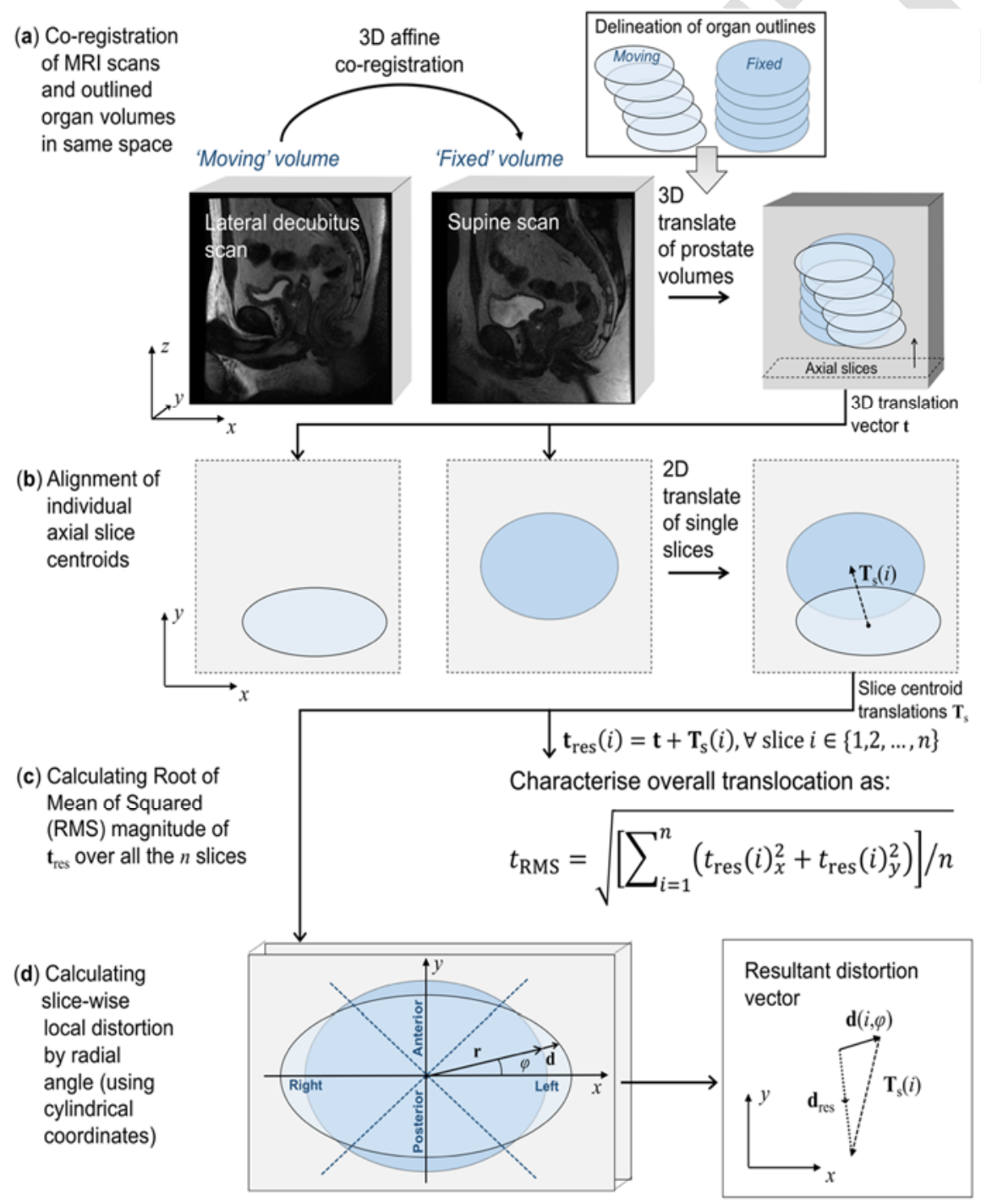


Fig. 2. Example distortion maps of two patients. (a,b) An example of negative distortion in the lateral part of the apex and mid-gland; (c,d) little apparent distortion. The fixed and moving volumes are depicted in the first and second columns, respectively. In order to show the slice section difference as well as the local translation, the 'tree' of slice centroid translations $\mathbf{T}_{\mathrm{s}}$ and the distortion surface map (along with the corresponding color map expressed in $\mathrm{mm}$ ) are shown in the third and fourth (fifth) columns, respectively.

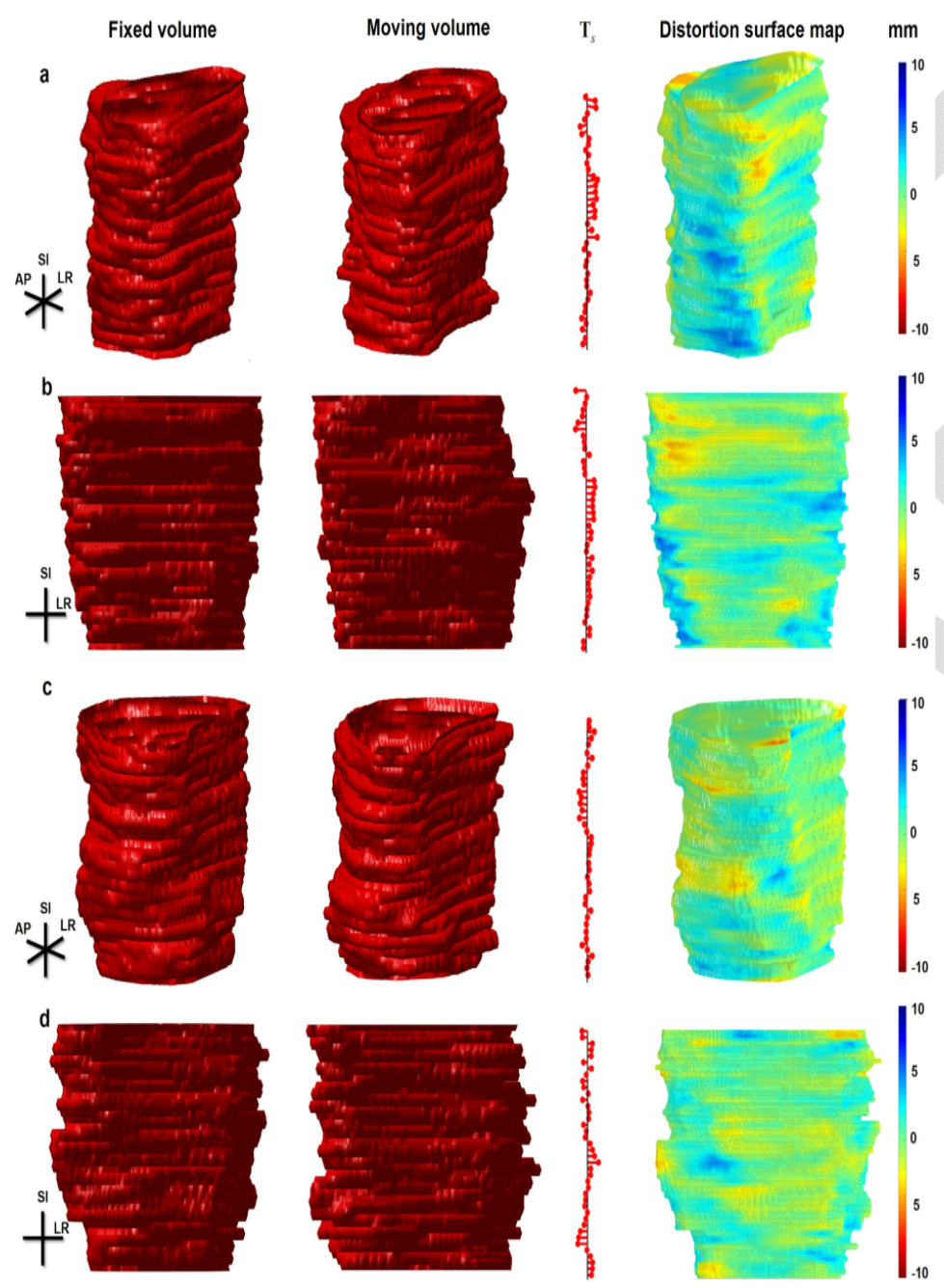


Fig. 3. Boxplot charts depicting (a) translocation of prostate and prostatic segments; and (b) direction of prostate translocation. The black solid line and the grey star marker denote the median and mean values, respectively.
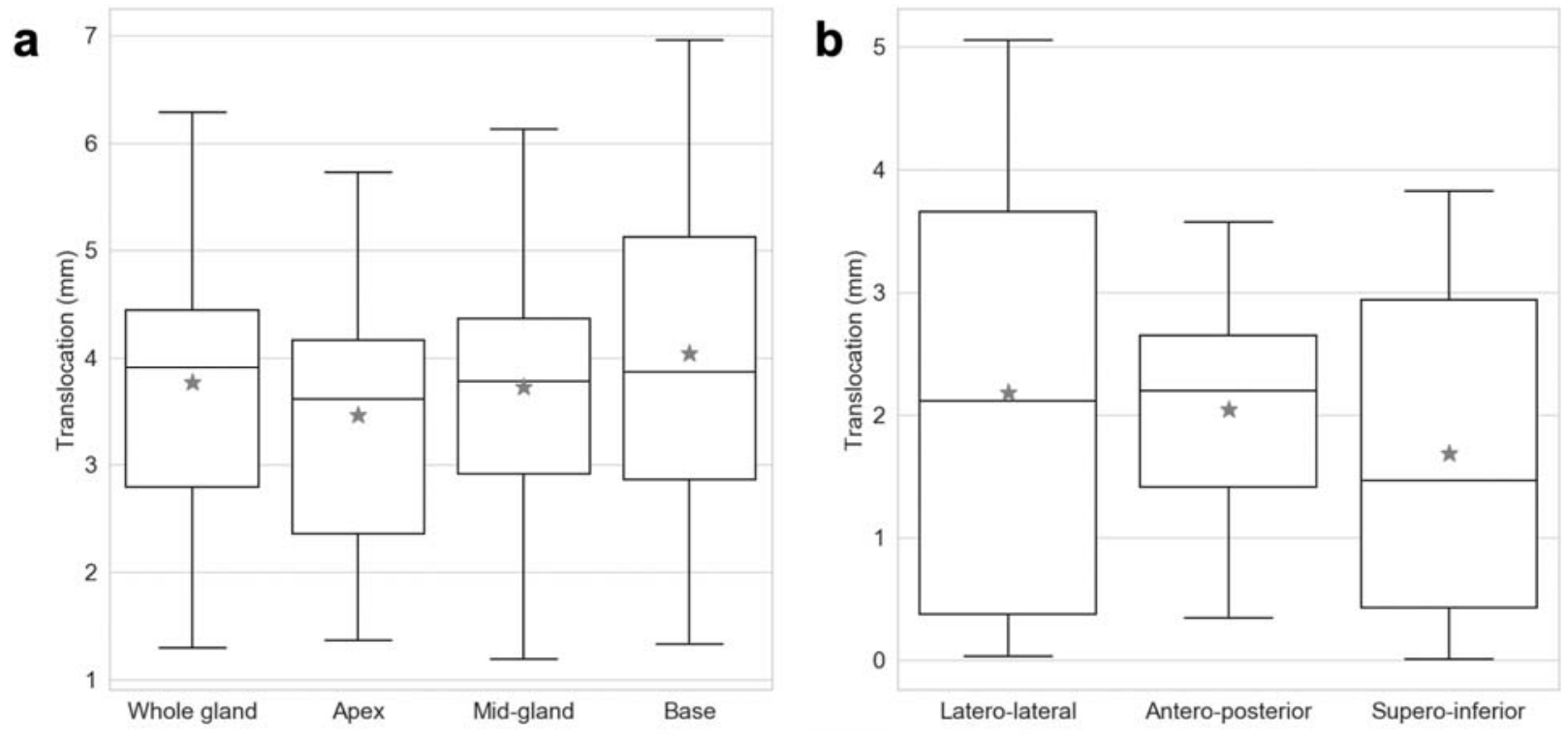
Fig. 4. Scatter plots of the translocation directions with relative volume difference. (a) Significantly moderate positive correlation in antero-posterior direction. (b) Moderate negative trend in latero-lateral direction.

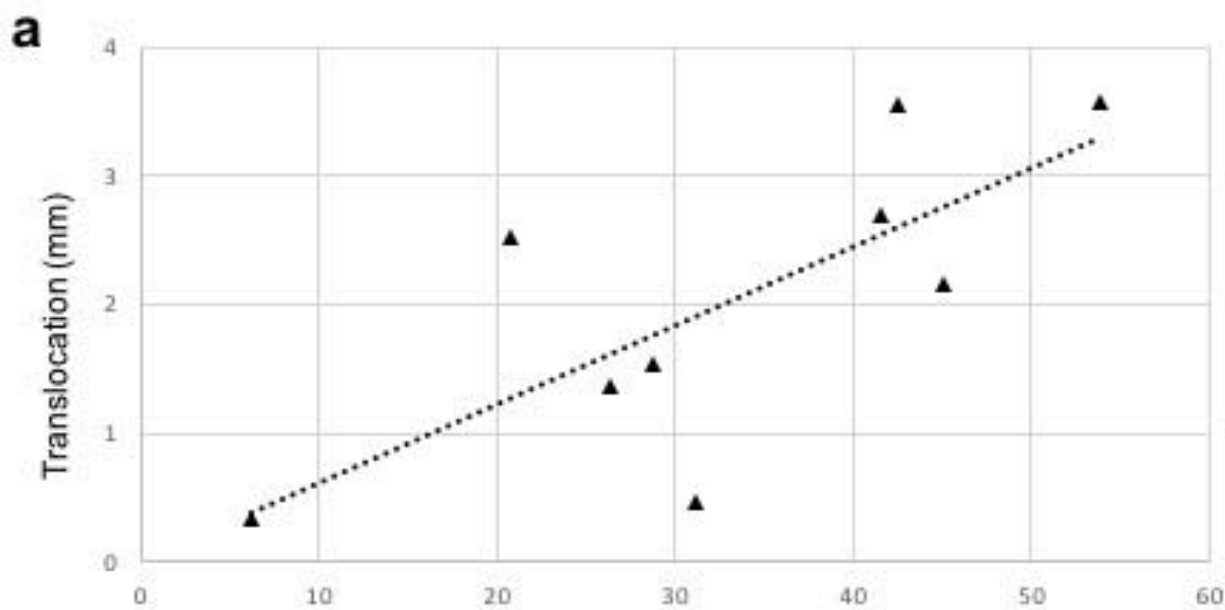

b Relative bladder volume difference (\%)

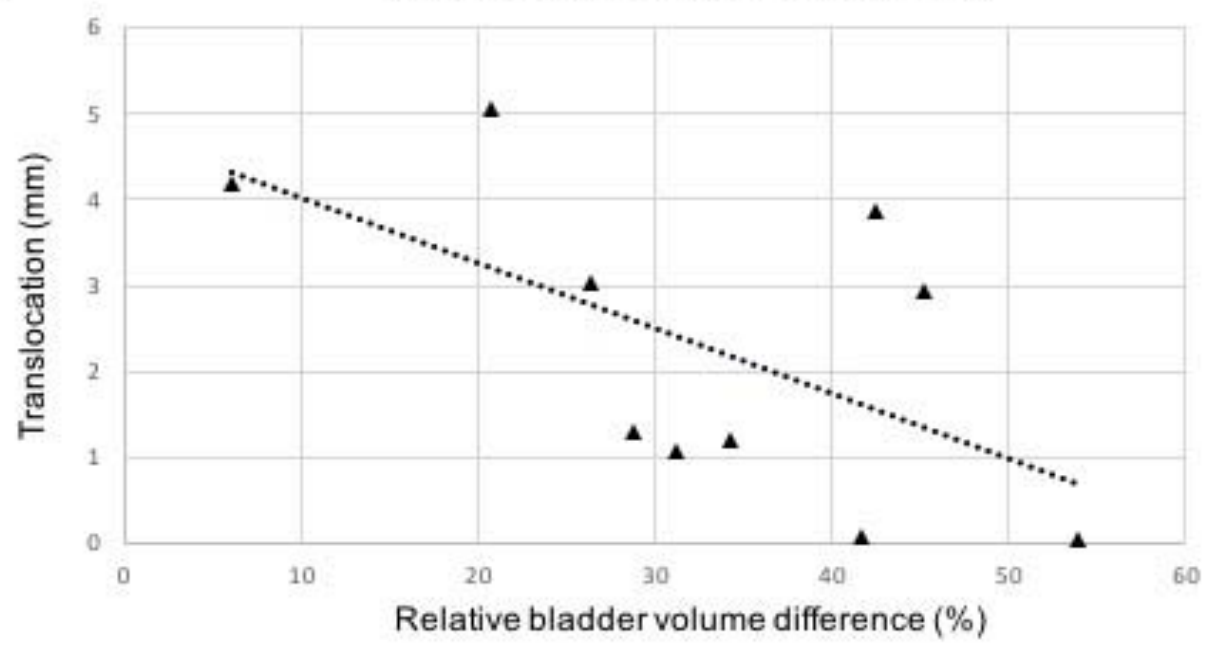




\begin{tabular}{|c|c|c|}
\hline & Mean \pm SD $(\mathbf{m m})$ & $9^{\text {th }}$ percentile $\pm S D(\mathrm{~mm})$ \\
\hline Whole gland & $1.78 \pm 0.39$ & $3.20 \pm 0.64$ \\
\hline Apex & $1.63 \pm 0.43$ & $2.78 \pm 0.71$ \\
\hline Mid-gland & $1.83 \pm 0.38$ & $3.20 \pm 0.64$ \\
\hline Base & $1.87 \pm 0.52$ & $3.44 \pm 0.96$ \\
\hline Prostate, anterior & $1.68 \pm 0.38$ & $3.02 \pm 0.65$ \\
\hline Prostate, posterior & $1.73 \pm 0.43$ & $3.02 \pm 0.75$ \\
\hline Prostate, left side & $1.85 \pm 0.44$ & $3.36 \pm 0.76$ \\
\hline Prostate, right side & $1.86 \pm 0.36$ & $3.36 \pm 0.59$ \\
\hline
\end{tabular}

\begin{tabular}{|c|c|c|}
\hline & $\begin{array}{c}\text { Translocation } \\
(\text { mean } \pm \text { SD })\end{array}$ & $\begin{array}{c}\text { Distortion } \\
(\text { mean } \pm \text { SD })\end{array}$ \\
\hline Whole gland & $0.64 \pm 0.18 \mathrm{~mm}$ & $0.79 \pm 0.12 \mathrm{~mm}$ \\
\hline Apex & $0.57 \pm 0.18 \mathrm{~mm}$ & $0.66 \pm 0.12 \mathrm{~mm}$ \\
\hline Mid-gland & $0.60 \pm 0.18 \mathrm{~mm}$ & $0.74 \pm 0.12 \mathrm{~mm}$ \\
\hline Base & $0.74 \pm 0.25 \mathrm{~mm}$ & $0.92 \pm 0.17 \mathrm{~mm}$ \\
\hline
\end{tabular}




\section{Appendix}

Let $\mathbf{t}$ be the vector defining the translation between the centres-of-mass of the two prostate glands under investigation. In addition, relying on this 3D rigid-body (translation alone) volume alignment, the centroids of the slice delineations were computed, by means of a slice-by-slice approach, according to the 'fixed' image space (rightmost part of Figure 1a). Afterwards, the slice delineations concerning the 'moving' volume were translated onto the 'fixed' image space so that the two centroids were coincident (Figure 1b). These slice-wise translations were stored in the $\mathbf{T}_{\mathrm{s}}(i)$ data structure, for each slice $i \in\{1,2, \ldots, n\}$.

With regard to the prostate distortion evaluation, let $\mathbf{d}(i, \varphi)$ denote the local radial distortion vector for the current slice $i \in\{1,2, \ldots, n\}$ and the angle $\varphi$ by computing the $(x, y)$ radial vector from the 'moving' slice outline to the 'fixed' slice outline, evaluated at an interval $\delta \varphi$ in radial angle from the common centroid point. As a matter of fact, since the slice thickness was typically much higher than the in-plane pixel size, the local distortion was suitably assessed in cylindrical coordinates rather than in spherical coordinates. ${ }^{20}$

In our analysis, a subdivision into thirds (i.e., apex, mid-gland, and base) was considered by sequentially assigning $\lfloor N / 3\rfloor$ slices to each region when $[N / 3\rfloor \equiv 0(\bmod 3)$, where $\lfloor\cdot\rfloor$ is the floor operator. In the case of $\lfloor N / 3\rfloor \equiv 1(\bmod 3)$, the remaining slice is assigned to the midgland. In the case of $\lfloor N / 3\rfloor \equiv 2(\bmod 3)$, each of the two remaining slices is assigned to the midgland and base regions, respectively.

\section{Prostate translocation}

Hereby, we considered the 'resultant translocation' $\mathbf{t}_{\text {res }}(i)$ as the vector addition of $\mathbf{t}_{2 \mathrm{D}}$ (denoting the $x$ and $y$ coordinates of the translation vector $\mathbf{t})$ and $\mathbf{T}$, so providing a measure of the global translocation of the prostate (see Figure 1c):

$$
\boldsymbol{t}_{\text {res }}(i)=\boldsymbol{t}+\boldsymbol{T}_{s}(i), \forall i \in\{1,2, \ldots, n\} .
$$

Aiming at achieving a comprehensive measurement of the total mean prostate gland translocation over the whole organ, the Root Mean Square (RMS) value of the magnitude of the resultant translocation vector $\mathbf{t}_{\text {res }}$ was calculated by averaging over all the $n$ slices according to Eq. (2): 


$$
t_{R M S}=\sqrt{\left[\sum_{i=1}^{n}\left(t_{r e s}(i)_{x}^{2}+t_{r e s}(i)_{y}^{2}\right)\right] / n}
$$

Along with the whole prostate gland, this calculation was performed also for the three thirds to highlight the different contributions in the three prostate regions.

\section{Prostate distortion}

In order to better characterise the prostate distortion, the 'resultant distortion' $\mathbf{d}_{\text {res }}$ (slice, $\varphi$ ) was computed from the slice-wise vector addition of $\mathbf{d}$ and $\mathbf{T}_{\mathrm{s}}$, so gaining insights into the combined effects of translational and local distortions (Figure 1d):

$$
\begin{gathered}
\boldsymbol{d}_{\text {res }}(i, \varphi)=\boldsymbol{d}(i, \varphi)+\boldsymbol{T}_{s}(i), \\
\forall i \in\{1,2, \ldots, n\}, \varphi \in\left\{k \cdot \delta \varphi, \text { withk }=0,1, \ldots, N \mid N \cdot \delta \varphi=360^{\circ}\right\},
\end{gathered}
$$

where $n$ and $N$ are the number of the slices and the number of radial angle intervals $\delta \varphi$ (used in the cylindrical polar coordinate system), respectively. In our case, we considered unitary angle increments (i.e., $\delta=1$ and $N=360$ ).

In addition to the measurements taking into account all the radial angles, for better appreciating the distortions' directions in the axial section, a subdivision of the prostate (considering the axial plane) into four quadrants, namely: anterior, posterior, left, and right.

At the end of this process, we considered descriptive statistics to summarize the results. In particular, the mean and the $90^{\text {th }}$ percentile (less affected by outliers than the maximum) were calculated over all the $n$ slices and the $N$ angle increments. 